\title{
Ensayo de evaluación continuada en la asignatura de Prótesis Estomatológica I
}

\section{Continuous assessment test in the Stomatological Prosthetic subject I}

EMILIO JIMÉNEZ-CASTELLANOS BALLESTEROS

ORCID: https://orcid.org/0000-0002-4275-8288

Universidad de Sevilla

Facultad de Odontología

Departamento de Estomatología

ejcb@us.eSs

Fecha de Recepción: 24-06-2019

Fecha de Aceptación: 05-07-2019

DOI: http://dx.doi.org/10.12795/9788447221912.044

Pр.: 1001-1023 


\section{Resumen}

El CIMA (Ciclos de Mejora en el Aula) que hemos desarrollado en el presente curso académico 2018-19, se ha llevado a cabo en la asignatura de Prótesis Estomatológica I de segundo curso del Grado de Odontología, básicamente consiste en, partiendo de la introducción de la gamificación como método de aprendizaje puesto en marcha en el curso anterior, incorporar una evaluación optativa continuada de la asignatura, como alternativa a la evaluación convencional mediante examen escrito final.

La experiencia ha sido muy gratificante, permitiendo una dinamización de la clase teórica que me era impensable conseguir al inicio de la actividad, es preciso destacar, que dicha actividad es imposible de llevar a cabo sin la participación activa de los alumnos, lo que supone un hándicap dada la habitual pasividad de los mismos en su trayectoria discente tanto a nivel de estudios preuniversitarios, como universitarios del Grado. Los resultados obtenidos demuestran, tanto el mayor grado de aprendizaje del alumno, como su mayor satisfacción con el procedimiento de aprendizaje y especialmente con la evaluación continuada, si bien hay discrepancias en la calificación final obtenida con respecto a la calificación del examen teórico. Es mi intención seguir esta trayectoria en cursos sucesivos, planteado el reto de estimular a los alumnos para conseguir una mayor participación en su aprendizaje.

Palabras Claves: Prótesis Estomatológica I, Grado en Odontología, Docencia Universitaria, Experimentación docente Universitaria, Evaluación continuada.

Jornadas de Formación e Innovación Docente del Profesorado | № 2 (2019) Esta obra se distribuye con la licencia Creative Commons 


\section{Summary}

The CIMA (Improvement Cycles in Classroom-ICIC) that we have developed in this school year 2018-19, has been carried out in the Stomatological Prosthetics I subject of the second year of the Degree in Dentistry, basically it was through gamification by Kahoot as instrument for interactive classes, incorporation of a continuous elective assessment, as an alternative to conventional evaluation (final written exam).

The experience has been very rewarding, allowing a revitalization of the theoretical class that I was unthinkable to get at the beginning of the activity, it should be noted that this activity is impossible to carry out without the active students join, which is a handicap in pre-universitary studies. The results show the best satisfaction with the learning process and especially continuous assessment. There are discrepancies between the final grade and the written exam. It is my intention to follow this trajectory in future courses, with the challenge of encouraging students to achieve greater participation in their learning.

Keywords: Stomatological Prosthesis I, Degree in Dentistry, University teaching, University teaching experience, Continuous assessment.

Jornadas de Formación e Innovación Docente del Profesorado | № 2 (2019) Esta obra se distribuye con la licencia Creative Commons 


\title{
Breve descripción del contexto
}

La asignatura de Prótesis Estomatológica I se imparte en el segundo semestre del segundo curso del Grado en Odontología. Tiene una carga lectiva de 6 créditos totales, 3 teóricos y 3 prácticos de laboratorio. La asignatura se imparte en dos grupos de teoría y dos de prácticas, siendo responsable en el presente curso académico del grupo B, con un total de 44 alumnos matriculados. El CIMA se aplicó en los contenidos teóricos de la materia de manera que el primer día del curso, además de realizar la presentación de la asignatura, se explicó la opción de realizar una evaluación continuada voluntaria, pidiendo a los alumnos interesados que así lo manifestaran por escrito, comprometiéndose y participando un total de 31.

Finalmente mencionar que el aula de impartición tiene una capacidad para 43 alumnos, dotada con todos los medios técnicos necesarios para llevar a cabo la actividad.

\section{Diseño previo del ciclo de Mejora Docente}

\author{
Mapa de contenidos y problemas que \\ describan los aprendizajes deseables \\ y los núcleos de trabajo en el aula \\ El mapa de contenidos de la asignatura se refleja a \\ continuación:
}



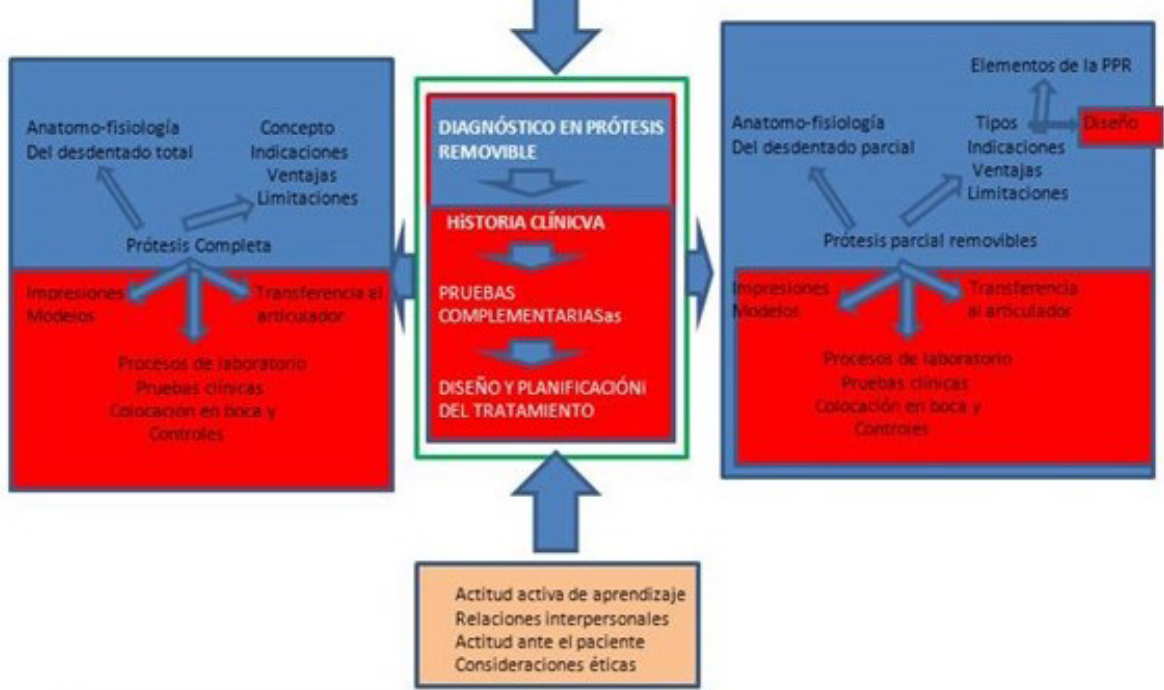

CONTENIDOS ESTRUCTURANTES

Contenidos conceptuales

Conteridos procedimentales

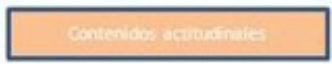

Figura 1. Contenidos de la asignatura

La asignatura se puede compendiar en tres grandes bloques temáticos:

1. ¿Cuál es la forma y función del Aparato Estomatognático y su aplicación al diagnóstico en Prótesis Estomatológica? (Contenidos cuadro superior)

2. ¿Cuáles son los procedimientos para el diagnóstico y tratamiento del desdentado total mediante prótesis removible? (Contenidos cuadro izquierdo)

Jornadas de Formación e Innovación Docente del Profesorado | № 2 (2019) Esta obra se distribuye con la licencia Creative Commons Reconocimiento-NoComercial-SinObraDerivada 
3. ¿Cuáles son los procedimientos para el diagnóstico y tratamiento del desdentado parcial mediante prótesis removible? (Contenidos cuadro derecho)

Los contenidos estructurantes $u$ organizadores se reflejan en el centro del mapa y hacen referencia a la realización de un correcto diagnóstico y plan de tratamiento en Prótesis removible estomatológica.

Estos contenidos estructurales incardinan el conocimiento de la anatomo-fisiología normal del Aparato Estomatognático para sentar las bases de las estructuras y funciones a rehabilitar en el paciente desdentado total mediante prótesis completa mucosoportada y en el paciente desdentado parcial mediante prótesis parcial removible dento, dento-muco o mucosoportada.

A su vez en cada uno de los sub-apartados o bloques se recogen los contenidos estructurantes de los mismos, (parte superior fisiología de la oclusión, parte izquierda prótesis completa, parte derecha prótesis parcial removible).

Por último, se han diferenciado en colores los contenidos conceptuales (a trabajar eminentemente en las clases teóricas) de los procedimentales (clases prácticas) y actitudinales (ambas).

\section{Modelo metodológico posible y secuencia de actividades programadas, partiendo de los problemas seleccionados}

\section{Preparación Previa}

Para trabajar las preguntas problemas, se dividieron por temas, de manera que se trabajaron tres temas en 
cada 2 horas de clase semanal, mediante la gamificación creé 12 "Kahoot" con un total de 10 preguntas cada uno (con una media de tres preguntas por tema) en dos formatos diferentes, en primer lugar en el formato "Survey", se dan cuatro respuestas posibles a la pregunta formulada, optando el alumno por la opción que le parezca más oportuna, pero sin apuntar la respuesta correcta, tan solo se refleja el porcentaje de alumnos que elije cada respuesta de las cuatro posibles.

Ejemplo de pregunta del tema 10:

¿Cuál es la finalidad del arco de transferencia en el dentado?

Respuestas:

Transferir relación del modelo superior respecto al cráneo

Transferir la distancia del modelo respecto al eje de giro

Transferir la inclinación del Plano de oclusión

Todas las anteriores

El siguiente tipo es en formato "Quiz" de similares características, pero aportando la solución final y la puntuación de los alumnos que tuvieran un mayor número de aciertos en el tiempo estipulado de respuesta. Este último formato me permitió además recoger, una vez finalizado "el juego", el porcentaje total de aciertos a las preguntas formuladas, así como la opinión "subjetiva" de los alumnos sobre su nivel de aprendizaje.

\section{Cuestionario inicial-final para hacer un seguimiento de la evolución de los estudiantes en relación con los problemas clave}

Las preguntas "problema” generales, con su escala de aprendizaje fueron las siguientes: 
1.- ¿Por qué el "Diagnóstico en Prótesis removible" aparece como eje central de la asignatura? (Se refiere al mapa de contenidos que se incluirá en el cuestionario)

Respuestas de menor a mayor:

a) No lo sé

b) Porque se relaciona con el resto de los contenidos

c) Porque es fundamental para saber la situación del paciente

d) Porque un correcto diagnóstico permitirá establecer una correcta indicación del Plan de tratamiento

\section{2.- ¿Cuál es la utilidad práctica en prótesis de conocer en profundidad la dinámica condilar y mandibular?}
a) no lo sé
b) no tiene ninguna utilidad
c) Para mejorar el tratamiento de los pacientes
d) Para conseguir prótesis con una anatomía oclu- sal compatible con la dinámica condilar del paciente

\section{3.- ¿Por qué es conveniente utilizar los articuladores semiajustables? \\ a) No sirven para nada \\ b) Para hacer prótesis \\ c) Para conseguir mejores resultados en nuestras prótesis \\ d) Para conseguir una correcta oclusión en las próte- sis y que sean funcionales}

\section{4.- ¿De qué depende la retención y estabilidad de una prótesis completa?}
a) De lo bien hecha que esté
b) De los rebordes alveolares residuales del paciente
c) De los rebordes alveolares residuales del paciente y del control muscular
d) De los rebordes alveolares residuales del paciente y del control muscular de la saliva y de la oclusión 
5.- ¿Por qué diseño una oclusión balanceada bilateral en prótesis completa?
a) no lo se
b) Porque es la más fácil de hacer
c) Porque mejora la estabilidad de la prótesis
d) Porque contribuye a mejorar la retención y estabi- lidad de la prótesis

\section{6.- ¿Cómo sé el tamaño y forma de dientes que debo indi- car en un paciente desdentado total?}
a) no lo se
b) Mirando fotos antiguas del paciente
c) Por los gustos del paciente
d) Por una serie de referencias faciales como son el labio superior en reposo, la línea de sonrisa y la línea de caninos.

\section{7.- ¿De qué dependerá que en un paciente desdentado parcial haga una prótesis esquelética o una prótesis par- cial de resina?}
a) No lo sé
b) De las preferencias y economía del paciente
c) Del diagnóstico
d) Del diagnóstico particularmente del número, dis- posición y superficie periodontal de los dientes remanentes

\section{8.- ¿Qué diferencia fundamental existe entre los tipos de prótesis de la pregunta anterior?}
a) No lo sé
b) el precio
c) los materiales de confección
d) La vía de transmisión de las fuerzas oclusales a través del diente o de la mucosa




\section{Aplicación del CIMA}

\section{Relato resumido de las sesiones}

\section{Introducción:}

El primer día de clase de impartición de la asignatura dedicada a explicar los objetivos, competencias, programa teórico y práctico, sistemas y criterios de evaluación, etc., al final de la misma, les puse dos pequeños videos de dos secuencias de películas muy conocidas. El primero de ellos era la secuencia de la película de Indiana Jones (en Busca del Arca perdida) en la que aparece Harrison Ford dando una clase de tipo "Magistral" con participación pasiva de los alumnos, en la segunda, El Club de los poetas muertos, Robin Williams da una clase con una participación y protagonismo de los alumnos. Tras el visionado de los mismos les pregunté a los alumnos ¿quiénes creían que habían aprendido más? A lo que mayoritariamente respondieron que los alumnos del segundo video.

Estos videos me sirvieron para obtener el compromiso de los alumnos para participar activamente en el CIMA, de manera que les pasé una lista, a modo de contrato, donde debían apuntarse si se comprometían a participar en el proceso de evaluación continuada, al tiempo que les suministré el cuestionario con las "Preguntas Problema" que sirvió, una vez analizado, para establecer una escalera inicial de aprendizaje.(compromiso inicial de 31 de los 44 alumnos matriculados).

\section{Sistema de Evaluación}

Las opciones de evaluación teórica fueron dos (recogidas en el Programa de la asignatura y aprobadas por el Departamento de Estomatología previo al inicio del curso): 
1. Alumnos que no participan en el CIMA: el clásico examen teórico final de 19 preguntas cortas (11 respuestas correctas para superar la materia).

2. Alumnos que participan en el CIMA: los alumnos participantes podrían obtener hasta 5 puntos de la calificación teórico final (que se sumaría a la calificación obtenida en el examen teórico final), estos 5 puntos se obtendrían de la siguiente forma:

1 punto por asistencia a todas las sesiones teóricas o con 1 falta

0,5 puntos por asistencia a todas las sesiones menos 2 faltas

0 si tuvieran más de dos faltas

De 0,1 a 1 punto en función de los 10 alumnos que obtuvieran la mayor puntuación en los Kahoot modelo Quiz (1 punto el de mayor puntuación, 0,9 el segundo y así sucesivamente)

Hasta 3 puntos en la resolución de casos problema correspondiente a los tres bloques temáticos que los alumnos trabajarían por grupos en tiempo "no presencial". En cada caso problema se plantean 10 preguntas que los alumnos deben contestar y que serán calificadas con 1 punto si es correcta y completa, 0,5 puntos correcta pero incompleta y 0 puntos incorrecta. Esta evaluación será hecha por el profesor y supondrá el $60 \%$ de los tres puntos referidos, el restante $40 \%$ se obtendrá de la evaluación por pares de los propios alumnos dentro de cada grupo mediante cuestionarios preeleborados.

En ambos casos la calificación final corresponde al 60\% para la evaluación teórica (Continua o clásica) y el $40 \%$ a la práctica (igual para todos los alumnos participantes o no en el CIMA, mediante examen práctico preclínico simulado). 
Secuencia de las sesiones:

La semana antes de la clase teórica, los alumnos tienen a su disposición los apuntes completos de cada uno de los temas a trabajar, así como las presentaciones con las imágenes ilustrativas de cada uno de los temas y el compromiso de leerlos y anotar las dudas que pudieran surgir, de la misma forma desde el primer día de clase los alumnos disponen del calendario completo de la asignatura donde se específica los temas y contenidos a tratar en cada sesión teórica y práctica.

Las sesiones de trabajo se han llevado a cabo como estaban diseñadas en el CIMA del pasado curso, es decir: a principio de la clase, dejamos 5 minutos para que los alumnos, solos o en grupos apuntaran todas las dudas referentes a los temas a tratar, seguidamente se realizó una puesta en común en la que los alumnos iban planteando sus dudas, siendo mi labor la de estimular y conducir la discusión para que fueran los propios compañeros los que contestaran las dudas propuestas, una vez resueltas, se procedió a realizar la primera fase de la gamificación, con los cuestionarios realizados en Kahoot con la modalidad "Survey", la cual da pie a establecer a su vez una discusión en el auditorio sobre cuál podría ser la respuesta correcta, ya que dicha aplicación tan solo señala el porcentaje de alumnos que han consignado cada una de las cuatros posibles respuestas. Es preciso destacar que las preguntas planteadas estaban especialmente diseñadas para poder conectar a su vez con otras cuestiones clave y permitir al mismo tiempo una visión integradora de la asignatura, para apoyar las explicaciones, cuando fue necesario, se dispuso de imágenes en Powerpoint y modelos reales de las cuestiones en discusión. Por último, a partir de la siguiente sesión y consecutivas, al inicio de cada una, se volvió a realizar la gamificación, pero esta vez con el modelo "Quiz" (que señala la respuesta correcta una vez participados los alumnos) junto a una escala de puntuación 
de los alumnos acertantes, lo que sirvió de repaso de los temas tratados en la sesión anterior. (Es preciso aclarar que las preguntas de ambos Kahoot en sus dos modalidades eran siempre las mismas, pero cambiadas de orden, tanto las preguntas como las respuestas, para evitar la "Memoria fotográfica"), el resto de la sesión continuaba como se ha descrito.

Por otra parte, se han incorporado clases de "Repaso" al final de cada uno de los bloques temáticos para intentar compendiar todo lo aprendido en las clases anteriores, así como un seminario final pre-examen, para resolver cuestiones y dudas finales. Dichas clases siguieron una dinámica similar a la planteada en el resto de las clases, es decir, la gamificación como herramienta de estímulo y orientación en el proceso de aprendizaje de los alumnos.

En relación a los grupos de trabajo, fueron asignados aleatoriamente a principio de curso con un máximo de cinco alumnos por grupo. Los trabajos asignados fueron un total de tres (correspondiente a cada uno de los bloques temáticos) y que los alumnos debían trabajar en su carga lectiva "No presencial", que eran colgados en la plataforma en fechas predeterminadas en el calendario anual de la asignatura, así como su fecha de entrega junto a una autoevaluación y evaluación por pares del trabajo de cada uno de los alumnos del grupo.

En lo referente al clima de trabajo, de un lado he de señalar mi propia satisfacción, pues una vez pasadas las primeras sesiones y la inquietud e incertidumbre sobre cómo se desarrollarían y si sería capaz o no de mantener un nivel alto de atención, pude comprobar para mi grata sorpresa, que la participación de los alumnos asistentes fue incrementándose progresivamente, no solo en cuanto a la libre formulación de opiniones sino en relación al nivel de aprendizaje que demostraban adquirir, teniendo que reconocer que yo mismo estaba deseoso de que llegara la 
siguiente sesión por lo bien que me lo he estado pasando en clase, disfrutando como hacía años que no lo hacía.

Es preciso destacar qué con el compromiso previo adquirido con los alumnos a principio de curso, el porcentaje de participantes en el CIMA se ha incrementado notablemente con respecto a la experiencia del curso anterior (70,5\% frente al escaso $11,32 \%$ del año anterior), solo un alumno de los 31 tuvo más de dos faltas a las sesiones ( 3 faltas).

Entre las dificultades encontradas podemos señalar las complicaciones técnicas sufridas en algunas sesiones en las que el acceso a internet fue imposible o se interrumpía secuencialmente, por otra parte algunos alumnos se quejaban que la velocidad de respuesta a los Kahoot, estaba en relación con la capacidad de sus dispositivos moviles o PC y por último señalar que al aproximarse las fechas de exámenes finales se palpaba en la clase una menor preparación de los temas por parte de los alumnos. A su vez, la participación en las discusiones de las distintas sesiones, es dispar, estando lideradas en general por un determinado grupo de alumnos, que se han correspondido con los que han obtenido mejores calificaciones finales, frente a otros que tenían una actitud más pasiva.

Aun no considerándola una dificultad insalvable, he de mencionar que dada la rigidez del calendario académico impuesto por la Universidad, la duración teórica del CIMA, de acuerdo a las normas del ICE, no coincide con la duración temporal de la asignatura, hasta tal punto, que el presente CIMA se inició al principio de la asignatura y no como consta en el cronograma propuesto en la memoria inicial, ya que entendí que según las normas establecidas el CIMA debía aplicarse a la asignatura completa y no a parte de ella. Por otra parte, una de las condiciones para participar en la REFID es la de incrementar los créditos dedicados al CIMA con respecto a la edición anterior, 
lo que es imposible una vez que se ha aplicado a la asignatura completa.

\section{Evaluación del aprendizaje de los alumnos}

Dada la complejidad de todo proceso de evaluación, he optado por realizar distintos procedimientos.

\section{Primero}

A través de la propia aplicación del Kahoot en su modelo "Quiz", en las doce sesiones realizadas pude obtener la información que se recoge en la tabla 1, junto a la puntuación media obtenida en cada una de ellas.

Tabla 1. Resultado de la información suministrada con el total de gamificaciones realizadas con el modelo "Kahoot Quiz".

\begin{tabular}{|c|c|c|c|c|}
\hline Información recogida & $\begin{array}{c}\text { Puntuación } \\
\text { media }\end{array}$ & $\begin{array}{l}\text { Desviación } \\
\text { estándar }\end{array}$ & mínimo & máximo \\
\hline $\begin{array}{l}\text { Porcentaje de } \\
\text { respuestas correctas } \\
\text { de los alumnos del } \\
\text { total de “Kahoot" ( } 120 \\
\text { preguntas totales) }\end{array}$ & $86,70 \%$ & 14,190 & $52,22 \%$ & $95,48 \%$ \\
\hline $\begin{array}{l}\text { Porcentaje de } \\
\text { respuestas incorrectas } \\
\text { de los alumnos del } \\
\text { total de "Kahoot" (120 } \\
\text { preguntas totales) }\end{array}$ & $13,29 \%$ & 14,190 & $4,52 \%$ & $47,78 \%$ \\
\hline $\begin{array}{l}\text { Grado de "diversión } \\
\text { del alumno" con "La } \\
\text { gamificación" ( } 0 \text { a 5) }\end{array}$ & 3,66 & 0,644 & 2,5 & 4,4 \\
\hline $\begin{array}{l}\text { Grado de "aprendizaje } \\
\text { subjetivo del alumno" } \\
\text { con "La gamificación" } \\
\text { (\%) }\end{array}$ & $71,51 \%$ & 27,01 & $25 \%$ & $100 \%$ \\
\hline
\end{tabular}

Jornadas de Formación e Innovación Docente del Profesorado | № 2 (2019) Esta obra se distribuye con la licencia Creative Commons Reconocimiento-NoComercial-SinObraDerivada 
¿Recomendaría el

alumno este sistema?

(\%)

$78,93 \%$

22,07

$40 \%$

$100 \%$

Sensación positiva de

satisfacción del alumno

tras las sesiones (\%)

$52,46 \%$

25,85

$0 \%$

$80 \%$

Sensación neutra de

satisfacción del alumno

tras las sesiones (\%)

$30,11 \%$

18,85

$0 \%$

$60 \%$

Sensación negativa de

satisfacción del alumno

tras las sesiones (\%)

$22,17 \%$

20,71

$0 \%$

$55,56 \%$

№ de alumnos

participantes en las

sesiones

30,08

1,165

28

31

De estos resultados podemos destacar el alto porcentaje de respuestas correctas a las preguntas de los Kahoot (86,70\% de respuestas correctas de todos los alumnos a las 120 preguntas), lo que coincide con la alta sensación "subjetiva" de aprendizaje del alumno (71,51\%), destacar que un $78,93 \%$ de los alumnos recomendaría la gamificación como ayuda en el proceso de enseñanza/aprendizaje.

Por último, señalar que de los diez alumnos que puntuaron en la evaluación continuada en los Kahoot, salvo dos se correspondieron con las calificaciones más altas obtenidas en el examen teórico.

\section{Segundo}

El segundo procedimiento se llevó a cabo comparando el cuestionario inicial con el final, para establecer la evolución de las "Escaleras de aprendizaje", no obstante, solo se consignan los resultados de los alumnos que participaron en los dos cuestionarios $(n=25)$ (no considerando los seis alumnos que o no realizaron ninguno de los dos cuestionarios o solo uno de ellos).

Jornadas de Formación e Innovación Docente del Profesorado | № 2 (2019) Esta obra se distribuye con la licencia Creative Commons 
En la tabla 2 se recoge el análisis descriptivo (escalón mínimo, escalón máximo, media y desviación estándar) de los escalones de aprendizaje de cada una de las 8 preguntas del cuestionario, de manera comparativa entre las respuestas iniciales y las finales (de 1 a 4 escalones en cada pregunta).

Tabla 2. Análisis descriptivo de las escaleras de aprendizaje correspondientes a los cuestionarios incial/final de los alumnos evaluados.

\section{Estadísticos descriptivos}

\begin{tabular}{lcccrc} 
& N & Mínimo & Máximo & Media & $\begin{array}{r}\text { Desviación } \\
\text { estándar }\end{array}$ \\
\hline primera pregunta inicial & 25 & 1 & 4 & 1,20 &, 707 \\
primera pregunta final & 25 & 1 & 4 & 2,44 & 1,446 \\
segunda pregunta inicial & 25 & 1 & 4 & 1,40 &, 957 \\
segunda pregunta final & 25 & 1 & 4 & 3,44 & 1,003 \\
tercera pregunta inicial & 25 & 1 & 4 & 1,64 &, 995 \\
tercera pregunta final & 25 & 1 & 4 & 2,68 & 1,069 \\
cuarta pregunta inicial & 25 & 1 & 1 & 1,00 &, 000 \\
cuarta pregunta final & 25 & 1 & 4 & 2,36 &, 860 \\
quinta pregunta inicial & 25 & 1 & 3 & 1,32 &, 748 \\
quinta pregunta final & 25 & 1 & 4 & 2,68 &, 945 \\
sexta pregunta inicial & 25 & 0 & 2 & 1,04 &, 351 \\
sexta pregunta final & 25 & 0 & 4 & 3,40 & 1,155 \\
séptima pregunta inicial & 25 & 1 & 2 & 1,12 &, 332 \\
séptima pregunta final & 25 & 1 & 4 & 3,20 & 1,080 \\
octava pregunta inicial & 25 & 1 & 4 & 1,80 &, 816 \\
octava pregunta final & 25 & 1 & 4 & 3,33 &, 868
\end{tabular}

Podemos observar como se ha producido un incremento en todas las preguntas en los escalones de 
aprendizaje a favor del cuestionario final, que oscila desde 1 a 2,5 escalones promedio.

A su vez se realizó un análisis inferencial para determinar si existían diferencias significativas entre los escalones de aprendizaje de las respuestas iniciales/finales, mediante el test de Wilcoxon para muestras apareadas, resultando todos ellos significativos para un valor de $p<.01$.

Tercero

Por último, queríamos proceder a comprobar la calificación final obtenida en el examen teórico y práctico final de la asignatura por los alumnos que habían asistido a las sesiones de trabajo $(n=31)$, comparada con la de aquellos alumnos que no participaron en el CIMA, sin embargo, de los alumnos que no realizarón la evaluación continuada solo se presentaron al examen escrito final dos y solo uno de ellos superó la materia. De los alumnos de la evaluación continuada, se presentaron todos, 18 superaron el examen teórico $(58,06 \%)$, pero con el sistema de evaluación continuada todos ellos aprobaron la materia.

\section{Evaluación del CIMA puesto en práctica}

La evaluación del CIMA se realizó a través de un cuestionario de satisfacción que se pasó a los alumnos en la última sesión, cuyo resultado podemos consultar en las tablas 4 y 5.

Jornadas de Formación e Innovación Docente del Profesorado | № 2 (2019) Esta obra se distribuye con la licencia Creative Commons Reconocimiento-NoComercial-SinObraDerivada 
Tabla 4. Resultados del cuestionario de satisfacción de los alumnos, de las preguntas del cuestionario puntuadas de 0 a 5

$\begin{array}{lllll}\begin{array}{l}\text { Pregunta del cuestionario } \\ \mathrm{n}=31\end{array} & \begin{array}{l}\text { Puntuación } \\ \text { media }\end{array} & \begin{array}{l}\text { Desviación } \\ \text { 0 a } 5 \text { puntos }\end{array} & \text { estándar } & \text { Valor } \\ & \text { mínimo } & \text { máximo }\end{array}$

¿Qué puntuación le

pondrías al estilo de clase

habitual que te imparten

en otras asignaturas?

¿Qué puntuación le

pondrías al estilo de clase

interactiva que hemos

seguido en la asignatura

de prótesis?

Evalúa lo que crees que

has aprendido en la

Clase habitual en otras

asignaturas

Evalúa lo que crees que

has aprendido en la Clase

Interactiva que hemos

seguido en la asignatura

de prótesis

¿Qué puntuación le

pondrías a la calificación

de la asignatura de forma

clásica tan solo con un

examen teórico?

¿Qué puntuación le

pondrías a la calificación

de la asignatura mediante

evaluación continuada

además del examen

teórico?
3,23

, 920

1

5

3,42

1,148

1

5

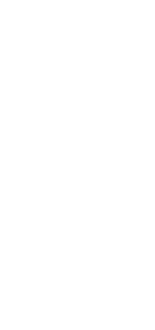

Podemos observar como en todos los casos las puntuaciones medias de la "Clase interactiva" supera a la "Clásica", destacando en particular la diferencia en lo relativo a la evaluación, de 1,84 (DS1,157) para la evaluación

Jornadas de Formación e Innovación Docente del Profesorado | № 2 (2019) Esta obra se distribuye con la licencia Creative Commons Reconocimiento-NoComercial-SinObraDerivada Internacional (CC BY-NC-ND 4.0.) 
clásica (solo mediante examen teórico final), frente a un 4,71 (DS0,529) del modelo de evaluación continuada propuesto en este CIMA.

Sobre estos resultados se realizó un análisis inferencial para determinar si existían diferencias significativas entre las preguntas pareadas, (que comparaban ambos tipos de clase), es decir, la 1 con la 2; la 3 con la 4 y la 5 con la 6 (comparan los dos tipos de evaluación), mediante el test de la t de Student para muestras relacionadas, resultando significativas, a favor de la clase interactiva la comparación 3 y 4 ; y a favor de la evaluación continuada (la comparación 5 y 6) para una $\mathrm{p}<.01$. No existíendo diferencias entre la 1 y 2.

Tabla 5. Resultados del cuestionario de satisfacción de los alumnos de las preguntas del cuestionario de elección entre "Clase magistral clásica" y "Clase interactiva".

\begin{tabular}{|c|c|c|}
\hline $\begin{array}{l}\text { Pregunta del cuestionario } \\
n=31\end{array}$ & $\begin{array}{l}\text { Clase magistral clásica } \\
\text { Frecuencia/Porcentaje }\end{array}$ & $\begin{array}{l}\text { Clase inter } \\
\text { Frecuencvia }\end{array}$ \\
\hline $\begin{array}{l}\text { Aunque a final de curso } \\
\text { tuvieras la máxima } \\
\text { calificación, si pudieras } \\
\text { elegir, ¿qué tipo de clase } \\
\text { de las dos te gustaría } \\
\text { tener? }\end{array}$ & 9 / 29\% & $22 / 71 \%$ \\
\hline $\begin{array}{l}\text { ¿Cuál de las dos clases te } \\
\text { ha resultado más amena? }\end{array}$ & 4 / 12,9\% & $26 / 83,9 \%$ \\
\hline $\begin{array}{l}\text { ¿En cuál de las dos clases } \\
\text { has tenido que trabajar } \\
\text { más? }\end{array}$ & 7 / 22,6\% & $24 / 77,4 \%$ \\
\hline
\end{tabular}

Podemos observar como en las tres preguntas formuladas el porcentaje de alumnos que opta por la clase interactiva es abrumadoramente superior (71\%), resultándole además más amena (83,9\%), a pesar de que reconocen que deben trabajar más en este tipo de clases (77,4\%). 
Según el modelo de evaluación clásico (examen escrito) el pasado curso no superaron la materia el 41,17 \% de los alumnos matriculados (incluidos suspensos o no presentados), con este tipo de evaluación han superado la materia el 77,5\%, y de los 13 que no la superaron, 12 de ellos fue porque no se presentaron al exmane final.

Por último es preciso señalar que de los 31 alumnos comprometidos con el CIMA solo uno alumno tuvo tres faltas $(3,22 \%)$, otro 2 faltas $(3,22 \%)$ y ocho 1 falta $(25,8 \%)$ el resto asistieron a todas las sesiones (67,74\%).

\section{Cuestiones a mantener y cambios a introducir}

Las cuestiones a mantener son todas las referentes al desarrollo de las sesiones de trabajo, es decir, la estructura de la clase y la incorporación de la gamificación como instrumento de aprendizaje, incluido el hecho de incorporar el Kahoot modelo "Quiz" en la sesión siguiente, por los buenos resultados obtenidos, así como las "Clases de repaso" y el seminario pre-examen.

De la misma forma se mantendrá la opción de evaluación continuada, con algunas correcciones, para diferenciar entre los alumnos que han participado activamente de los que se han limitado a un mínimo esfuerzo.

a) La sistencia a las sesiones del CIMA se puntuara com 1 punto si asiste a todas las sesiones, 0,5 si tiene una falta y 0,25 si tiene dos,un númerto mayor de 3 faltas supondrá la automática eliminación del proceso de evaluación continuada.

b) En la evaluación de los trabajos en grupo, disminuiré el procentaje de calificación del alumnop al $20 \%$, porque tienden a autoevaluarse con la máxima nota, frente al $80 \%$ del profesor.

c) Para sumar los puntos de la evaluación continuada al examen final, será necesario obtener un mínimo de 9 respuestas correctas de las 19 preguntas del examen final. 
Por último, aunque no depende de mi persona, solicitaría la adecuación de los plazos para el desarrollo del CIMA con el calendario de actividades docentes de la Universidad, así como eliminar la condición de aumentar los créditos del CIMA en el siguiente curso académico, para acceder a la REFID, cuando se ha desarrollado este en la asignatura completa.

Los principios didácticos que deben guiar el presente/ futuro se basan en el cambio de paradigma de la figura del Profesor como transmisor del conocimiento en un facilitador y orientador del proceso individual de aprendizaje del alumno, de manera que genere en el mismo una actitud autocrítica que le permita seguir aprendiendo a lo largo de toda su vida, así como modificar los sistemas de evaluación clásica centradas en un proceso puramente memorístico y no en el trabajo y aprendizaje real del alumno.

Jornadas de Formación e Innovación Docente del Profesorado | № 2 (2019) Esta obra se distribuye con la licencia Creative Commons 


\section{Referencias Bibliográficas}

Bain, K. (2004). Lo que hacen los mejores profesores universitarios. Valencia: Publicaciones Universidad de Valencia.

Cox KR, Ewan CE.(1982). La docencia en Medicina. Barcelona. Doyma.

Porlán, R. (2017). Enseñanza Universitaria, Cómo mejorarla. Madrid: Editorial Morata.

Jornadas de Formación e Innovación Docente del Profesorado | № 2 (2019) Esta obra se distribuye con la licencia Creative Commons Reconocimiento-NoComercial-SinObraDerivada 4.0 Internacional (CC BY-NC-ND 4.0.) 